\title{
ÉTUDE HISTOLOGIQUE DE L'OVAIRE DE PENAEUS PAULENSIS PÉREZ-FARFANTE, 1967
}

\author{
T. U. WORSMANN, S. R. BARCELOS \& A. G. FERRI
}

Faculdade de Medicina Veterinária da Universidade de São Paulo

\section{SYNOPSIS}

The histologic observations carried out in the ovary of the pink shrimp reveal, during the sexual cycle, which presents phases that may be identified also by the anatomical examination, three cell types beyond the follicle-cells. All the cells of the proliferation zone, in the various initial phases of the oogenesis, were named oogonia, because its distinction requires cytological studies. Observations about the peripheral bodies of the germinal cells prosecute by means of electron microscope and histochemical tecniques.

\section{INTRODUCTION}

Nous avons pu constater, au cours de nos études anatomiques (Worsmann et al., 1971), que les ovaires de la crevette $P$. paulensis présentent trois lobes: antérieur, moyen et postérieur. Ces organes, au cours de leur maturation, subissent des modifications de couleur et de développement qui ont permis d'établir quatre phases dans le cycle: 
Stade I: ovaires imatures;

Stade II: ovaires mûrissant;

Stade III: ovaires mûrs;

Stade IV: ovaires vides après 1'expulsion des ovules et au début d'un nouveau cycle.

Chacune de ces périodes, reconnues par l'examen externe de la femelle, peut être caractērisée par son aspect microscopique.

Dans ce travail nous présentons les résultats des observations histologiques et la description des cellules germinales durant 1'évolution cyclique des gonades féminines.

Une étude de ce qui a été écrit à ce sujet, nous rend compte d'un nombre variable de périodes qui furent établies dans le cycle de maturation des crevettes de la famille Penaeidae (Cummings, 1961; King, 1948; Oka \& Shirata, 1965; Rao, 1968; Renfro \& Brusher, 1964 et Shaikmahmud \& Tembe, 1961). D'autre part, la nomenclature utilisée pour les cellules germinales, au cours de l'ovogénèse, est très variable, fait remarqué aussi chez les mammifères par Ham (1963).

Malgrē les divergences entre les auteurs, ceux-ci sont d'accord pour dire que 1 'ovaire mûr présente, invariablement, des "corps périphériques" (Cummings, 1961; Hudinaga, 1942; King, 1948; Olguỉn-Palacios, 1968 et Rao, 1968). Rao (op. cit.), toutefois, ne les a pas décelé dans 1 'ovaire mûr de deux espèces de crevettes indiennes.

Les corps périphériques sont des structures du cytoplasme avec la conformation de petits bâtonnets qui se disposent à la périphérie des ovules mûrs. Ils ont été décrits par Hudinaga (1942), avec la dénomination de "substance gélatineuse" et furent signalés par King (1948), sous 1a désignation de "corps périphériques". Le rôle de ces structures est inconnu; Hudinaga (op. cit.) suggère qu'elles auraient par but produire une enveloppe autour de 1'ovule au moment de "1'extrusion", c'est à dire, 1'expulsion des ovules. 


\section{MATÉRIEL ET MÉTHODES}

Notre matériel d'étude a été constitué par 12 femelles répresentantes des stades I, II, III et IV, obtenues durant 1a pêche commerciale de crevettes à Itajaí, Santa Catarina.

La dissection des ovaires s'est accomplie suivant une technique qui a fait l'objet d'un article spécial (Worsmann \& Neiva, 1972); ensuite, des fragments de 2 à $3 \mathrm{~mm}$ d'épaisseur ont été prélevés sur le tiers initial du lobe postérieur, en les fixant pendant 24 heures au formol neutre de Lillie (McManus \& Mowry, 1965) et au liquide de Bouin (Lillie, 1954). La déshydratation a été faite à l'alcool absolu, l'eclaircissage au xylol et 1'inclusion en paraffine. Le matériel inclus a été coupé à $5 \mu$ d'épaisseur et les coupes ont été colorées par les techniques suivantes indiquées par Lillie (1954): hématoxyline-eosine, trichromique de Mallory et de P. Masson pour la mise en évidence du collagène, Verhöeff pour les fibres ēlastiques et Foot-Wilder pour les fibres réticulaires.

Nous avons encore effectué la mensuration des cellules germinales, employant 1'oculaire micrométrique Zeiss et considérant le plus large axe de 30 cellules de chaque type.

\section{RÉSULTATS}

L'ovaire de $P$. paulensis présente un parenchyme et un stroma.

Le stroma est constitué de cloisons fibreuses qui émannent d'une mince capsule conjonctive qui recouvre les organes. Dans ce tissue les méthodes spéciales révèlent des fibres collagènes et réticulaires, tandis que 1 'on ne trouve les fibres élastiques qu'à la paroi des vaisseaux. Par leurs propres caractères morphologiques, on distingue des fibrocytes et des hystiocites; des fibres musculaires n'ont pas été observées. 
Le parenchyme ovarien est formé de cellules germinales et de cellules foliculeuses.

Sur une coupe longitudinale l'ovaire montre la zone de prolifération, qui s'étend dans toute la longueur de l'organe. La zone de prolifération est un amas de petites cellules, avec un noyau central et arrondi; la chromatine est dense, parfois comme un délicat réseau, ou même vésiculeux. Le cytọplasme, d'aspect homogène, est acidophile avec forte tendence à la basophilie. La disposition de la chromatine nous rapelle des phases de la division mitotique.

Les cellules de la zone de prolifération peuvent être nommées ovogonies, considérant sous cette dénomination toutes les étapes initiales de 1'ovogénèse. On doit remarquer que les cellules moins développées de la zone de prolifération se situent immédiatement sous la capsule, òu s'alignent les unes à côté des autres, constituant 1'épithélium germinatif.

Partant de la zone de prolifération les cellules germinales se développent et subissent de successives modifications jusqu'à la maturité complète de l'ovule. Nous pouvons distinguer, dans le cycle de maturation, trois types de cellules germinales, entre lesquels existent, évidemment, tous les intermédiaires :
a) cellules germinales basophiles;
b) cellules germinales acidophiles;
c) cellules germinales acidophiles avec des corps péripheriques.

Le pourcentage des diverses catégories cellulaires est variable suivant les stades et nous rend possible leur identification microscopique.

Cellules germinales basophiles: ce sont des cellules arrondies, ovoïdes ou polyédriques, d'une taille variable de 8 à $120 \mu$ environ, qui se caractérisent par leur cytoplasme assez basophile. Le noyau, sphérique et central, se montre clair et vésiculeux, riche en nucléoplasme et contenant un fin réseau de filaments peu colorables. La chromatine est répartie en petits blocs disposés à côte de la membrane nucléaire ou autour des nucléoles dont le nombre est variable. Le cytoplasme est basophile et d'aspect granuleux; 
les granulations, toutefois, ne sont pas parfaitement individualisés (Fig. 1).

Cellules germinales acidophiles: ces cellules sont volumineuses et de conformation polyédrique irrégulière, d'une taille de 150 à $200 \mu$.

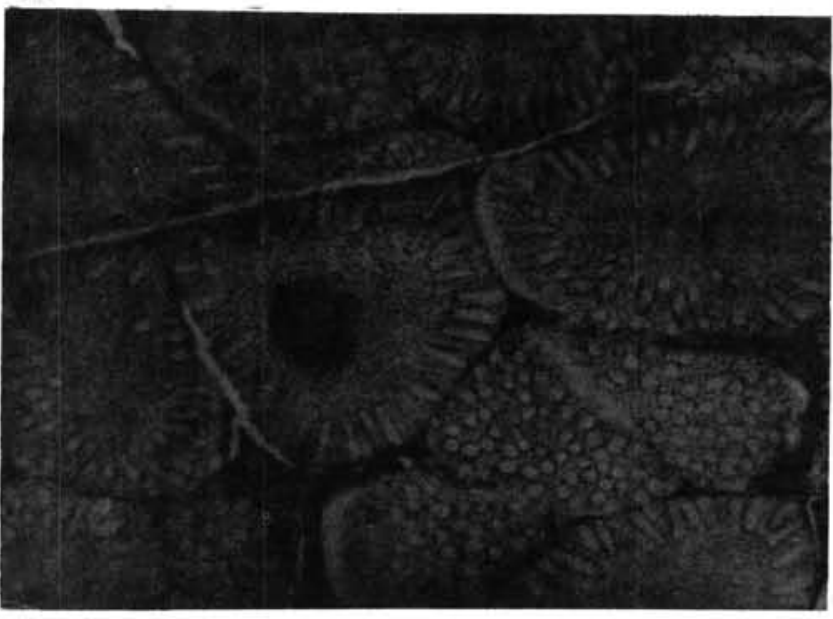

Fig. 1 - Cellules germinales basophiles. Hëmatoxylineéosine; X 480 .

E1les se caractērisent par leur cytoplasme chargē de gros grains sphēroïdes, assez acidophiles, réfringents et homogènes. Le noyau, arrondi, oval ou réniforme, occupe une position centrale et renferme un abondant nucléoplasme qui se précipite en fine granulation sous l'action des fixateurs utilisés. Il y a un à six nucléoles, généralement petits et hyalins, parfois volumineux, d'aspect homogène ou même granulaire. Les gros grains acidophiles sont immergés dans le hyaloplasme, qui se montre faiblement basophile et présente une disposition réticulaire lorsqu'il est examiné avec 1'objectif à immersion. Par la méthode de Mallory on distingue des grains se colorant en bleu et des grains se colorant en rouge, melangés et en proportions relatives variables. Un résultat similaire s'observe par le Masson, qui colore aussi le hyaloplasme en bleu, de sorte que la distinction des grains se fait par la différence de tonalité ou par la présence d'un mince et clair liséré autour d'eux (Fig. 2).

Cellules germinales acidophiles avec des corps péripheriques: ces cellules présentent des caractères morphologiques similaires à celles qu'on vient de décrire ci-dessus; elles sont, cependant, plus grandes, d'une taille de $350 \mu$ environ. 


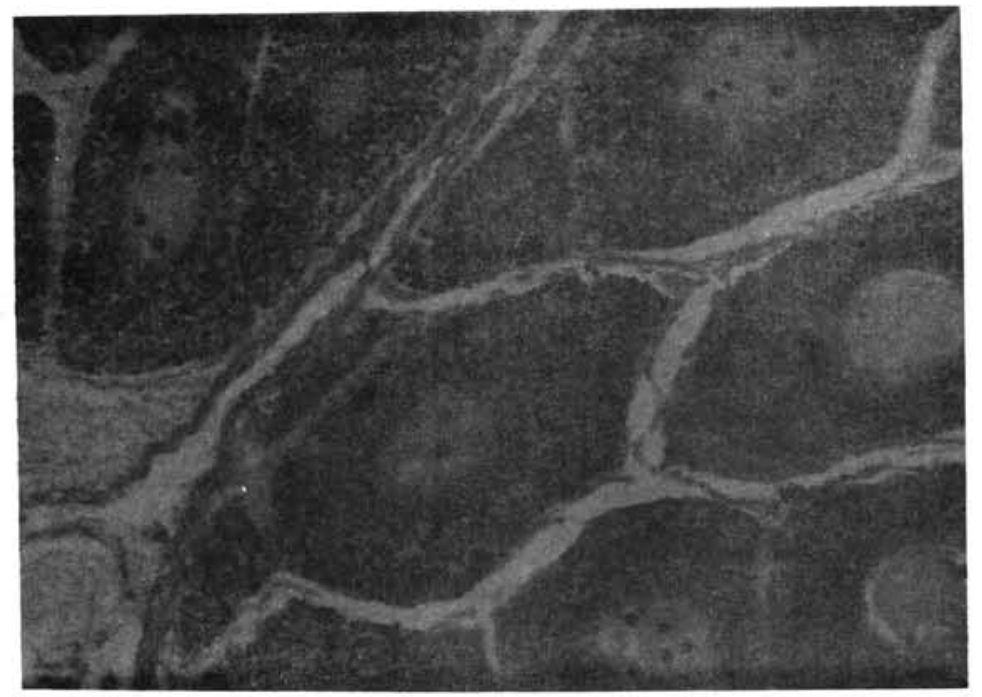

Fig. 2 - Cellules germinales acidophiles. Trichromique de Mallory; X 180.

Leur noyau souvent semble s'effacer, avec des limites imprécises, comme s'il y avait eu la dissolution de la chromatine dans le nucléoplasme; il semble que cet aspect résulte d'un phënomène dégéneratif, puisque, fréquemment, on rencontre des cellules remplies de vacuoles ou d'une substance amorphe et acidophile, et parfois entièrement desintegrées. D'autre part, la méthode de Masson révèle la prédominance de grains bleus clairs ou violacés, qui se colorent en châtain par le Mallory.

Néanmoins, ces cellules se caractērisent par 1a prēsence, dans la périphérie du cytoplasme, de larges corps avec la forme de bâtonnets. Ces corps, qui semblent subir un accroissement avec l'évolution de la maturation, se disposent les uns à côté des autres, radialement, laissant entre eux les grains cytoplasmiques, et sont apparemment adhérents à la membrane cellulaire. Ils ont un aspect homogène, sont colorables par l'eosine et manifestent une légère rétraction après les manipulations histologiques. Par 1a méthode de Masson les uns se colorent en bleu foncé, les autres en rouge; quelques uns prennent la double coloration. Ce même phénomène s'observe en employant la méthode Mallory, par laquelle ces structures se colorent en bleuâtre ou en rouge-orangé (Fig. 3).

Cellules foliculeuses: ce sont de petites cellules cubiques ou polyédriques dont les limites sont indistinctes. 


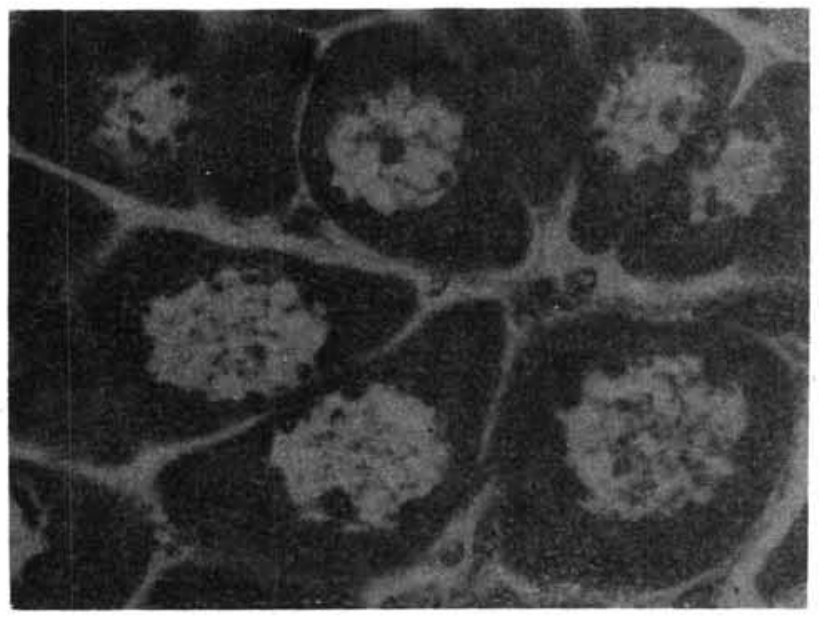

Fig. 3 - Cellules germinales acidophiles avec des corps périphëriques. Hëma toxylineéosine; X 150 .

Le cytoplasme est faiblement acidophile présentant une certaine basophilie; le noyau, arrondi ou oval, contient la chromatine en forme de délicate granulation. Ces cellules se situent sous la capsule, accompagnent les cloisons fibreuses et entourent, en une seule couche, les cellules germinales basophiles plus développées.

Dans les cellules foliculeuses qui entourent les cellules germinales acidophiles on observe que le noyau devient plus aplati et la chromatine plus dense, de sorte que, autour des cellules germinales acidophiles avec des corps périphériques, elles ont l'aspect d'un épithélium pavimenteux simple.

L'élimination des ovules conduit à la destruction du stroma, qui parfois est le siège de la dégénérescence hyaline; on rencontre aussi de diverses phases de dégénérescence tissulaire, surtout dans les cellules germinales résiduaires.

\section{DISCUSSION}

Nos résultats anatomiques (Worsmann et al., 1971) sont comparables à ceux obtenus dans les recherches poursuivies sur 1'ovaire d'autres espēces de crevettes (Cummings, 1961; King, 1948; Oka \& Shirata, 1965; 01guín- 
Palacios, 1968 et Rao, 1968), fait qui nous a permis 1'utilisation de la nomenclature établie par Rao (1968) pour nommer les différentes régions de 1 'organe.

Les auteurs ne sont pas d'accord en ce qui concerne le mombre de stades établis dans le cycle de maturation, prenant pour critère les modifications morphologiques présentées par les ovaires. Autant pour nos investigations anatomiques (Worsmann et al., 1971) que microscopiques, il nous a semblé préférable de diviser le cycle de maturation de $P$. paulensis en 4 périodes, caractérisées par $1^{\prime}$ existence de signes morphologiques facilement appréciables. Cette division, par ailleurs, est moins complexe, et peut être employée dans investigations relatives à l'établissement des époques de reproduction des crevettes dans nos différentes zones maritimes.

Le prélévement a été limité au tiers initial du lobe postérieur puisque des expériences préliminaires ont révelé que 1 'aspect microscopique de 1 'ovaire est le même dans tous les lobes (Worsmann et al., 1971); des observations identiques furent faites par Rao (1968) et King (1948).

Dans le stroma, les fibres élastiques ne sont trouvées qu'aux parois vasculaires, tandis que les fibres réticulaires, que nous avons mises en évidence, exigent de nouvelles études avec des techniques plus délicates, parce que nous n'avons pas réussi à couper le matériel par congélation. D'autre part, 1'absence de fibres musculaires a été aussi remarquée par King (1948) dans 1 'ovaire de Penaeus setiferus.

La zone de prolifération, décrite chez P. setiferus par Gutse11, d'après King (1948), observée par lui dans la même espèce, a été rencontrée chez $P$. paulensis, caractērisant 1 a région de 1 'organe où a lieu 1 'ovogénèse. Les phénomenes cellulaires qui s'y déroullent ne sont pas 1 'objet de notre recherche et demandent des connaissances plus spécialisées à cause de la diversité de terminologie (Gatz, cité par Ham, 1963). Nous avons donc englobé toutes les cellules dans les diverses phases initiales de 1 ' ovogénèse sous la dénomination de ovogonies.

La zone de prolifération est observée dans les quatre stades. À côté des cellules germinales basophiles elle répresente 1 'image histologique du stade I; la forte basophilie des cellules germinales suggère une intense activité de synthèse dans ces éléments. 
Le stade II est reconnu, à 1 ' examen microscopique, par la prédominance relative de cellules germinales acidophiles sur les basophiles, qui sont en petit nombre. Dans les cellules germinales acidophiles le cytoplasme fondamental, aperçu entre les grains qui le remplissent, conserve encore une certaine basophilie; cette granulation représente l'accumulation de vitellus dans le cytoplasme des cellules germinales.

On doit remarquer que, suivant Beams et Kessel (1963), la question de la vitellogénèse est assez discutable, divers mécanismes de vitellogénèse ayant èté décrits seulement chez les crustacés. Ces auteurs ont constaté, dans le crabe d'eau douce, un étroit rapport entre les éléments ergastoplasmiques et 1 'élaboration du vitellus.

Le groupement cellulaire dominant qui caractérise le stade III est constitué de cellules germinales acidophiles avec des corps périphériques, à côté d'un petit nombre de cellules germinales basophiles.

Suivant Hudinaga (1942), la plupart des auteurs prennent, comme signe de maturité, la présence de corps périphériques dans les ovules (Cummings, 1961; Hudinaga, 1942; King, 1948; Olguín-Palacios, 1968 et Rao, 1968). Rao (1968), à son tour, ne les a pas trouvé dans 1 'ovaire mûr de Metapenaeus affinis, Metapenaeus dobsoni et Parapeneopsis stylifera.

Dans la littérature spéciale nous n'avons pas obtenu de renseignement sur l'origine des corps périphériques. Hudinaga (1942) admet que ces structures renferment une substance gélatineuse; celle-ci, éliminée à la surface des ovules au moment de leur expulsion, formerait une enveloppe protectrice autour d'eux. D' après nos recherches, il semble que les corps périphériques sont liés à la membrane plasmatique; il faut, pourtant, de nouvelles recherches, surtout à l'aide du microscope électronique, pour que la génèse de ces éléments soit élucidée.

En ce qui concerne les cellules foliculeuses, il a été déjà signalé, par Hudinaga (1942), chez Penaeus japonicus, que ces éléments, initialement cubiques ou polyédriques, deviennent aplatis au cours de la maturation, par 1 'accroissement des cellules germinales.

Il semble que les cellules foliculeuses proviennent de l'épithélium germinatif, en accord avec l'opinion soutenue par plusieurs auteurs (King, 
1948; Porter \& Bonneville, 1968 et Zuckerman, 1962); toutefois, sans effectuer des études autoradiographiques on ne peut pas expliquer leur génèse, de même qu'addmetre l'existence d'un lignage cellulaire morphologiquement indistincte de 1'épithélium germinatif.

Finalement, on doit remarquer qu'à 1'époque de la ponte les femelles s'eloignent des régions exploitées par la pêche commerciale. Ainsi, les ovaires que nous avons recueillies étaient totalement vides, et il n'a pas été possible d'étudier le phénomène de l'expulsion des ovules. Cummings (1961) a aussi trouvé cet embarras au cours de ses recherches.

En conclusion de ce travail, la confrontation des résultats histologiques obtenus et des résultats anatomiques précédemment verifiēs (Worsmann et al., 1971), nous conduit à la reconnaissance de 4 stades, dans le cycle ovarian de $P$. paulensis, parfaitement caractérisées par des signes morphologiques facilement reconnaissables.

\section{RESUMO}

0 estudo histológico do ovário de Penaeus paulensis, nas diversas fases do processo de maturação do órgão, permitiu identificar três tipos de células germinativas, além de células foliculares.

Todas as células da zona de proliferação, que representam fases iniciais da ovogênese, foram englobadas sob a denominação genérica de ovogônias, uma vez que a sua distinção requer estudos citológicos. Estão sendo efetuadas investigações ultra-estruturais e histoquímicas à respeito dos corpos periféricos.

\section{BIBLIOGRAPHIE}

BEAMS, H. W. \& KESSEL, R. G. 1963. Electron microscope studies on developing crayfish oocytes with special reference to the origin of yolk. J. biophys. biochem. Cyto1., 18:621-651.

CUMMINGS, W. C. 1961. Maturation and spawning of the pink shrimp, Penaeus duorarum Burkenroad. Trans. Am. Fish. Soc., 90(4):462-468. 
HAM, A. W. 1963. Histologia. 2a. ed. Rio de Janeiro, Ed. Guanabara.

HUDINAGA, M. 1942. Reproduction, development and rearing of Penaeus japonicus Bate. Jap. J. Zoo1., 10(2):305-393.

KING, J. E. 1948. A study of the reproductive organs of the common marine shrimp, Penaeus setiferus (Linnaeus). Biol. Bull. mar. biol. Lab., Woods Hole, 94(3):244-262.

LILLIE, R. D. 1954. Histopathologic technic and practical histochemistry methods. 2nd ed. New York, Blakiston.

MAGALHÃES, E. F. 1943. Processo de determinação da maturidade do camarão. Bolm Minist. Agric. Ind. Com., Rio de J., 32(9):11-26.

McMANUS, J. F. A. \& MOWRY, R. W. 1965. Staining methods: histological and histochemical. 3rd ed. New York, Hoeber.

OKA, M. \& SHIRHATA, S. 1965. Studies on Penaeus orientalis Kishinouye. II. Morphological classification of the ovarian eggs and the maturity of the ovary. Bull. Fac. Fish. Nagasaki Univ., 18:30-40.

OLGUIN-PALACIOS, M. 1968. Estudio de la biología del camarón café Penaeus californiensis Holme. F.A.0. Fish rep., (57) 2:331-356.

PÉREZ-FARFANTE, I. 1967. A new species and two new subspecies of shrimp on the genus Penaeus from the western Atlantic. Proc. biol. Soc. Wash., 80:83-100.

PORTER, K. R. \& BONNEVILLE, M. A. 1968. Fine structure of cells and tissues. 3rd. ed. Philadelphia.

RAJYALAKSHMI, T. 1961. Studies on maturation and breeding in some estuarine palaemonid prawns. Proc. natn. Inst. Sci. India, ser. Biol. Sci., $27(4): 179-188$.

RAO, P. V. 1968. Maturation and spawning of the penaeid prawns of the southwest coast of India. F.A.0. Fish. Rep., (52) 2:285-302.

RENFRO, W. C. \& BRUSHER, H. A. 1964. Population distribution and spawning. Circ. Fish Wild1. Serv. Wash., (183):13-15.

SHAIKMAHMUD, F. S. \& TEMBE, V. B. 1961. A brief account of the changes in the developing ovary of Parapenaeopsis stylifera in relation to maturation and spawning cycle. J. Univ. Bombay, Biol. Sci., 29(3/5): $62-77$. 
WORSMANN, T. U. \& NEIVA, G. S. 1972. Técnica de necroscopia em camarão. Revta Med. vet., S Paulo, 7(3):259-267.

Contribuição ao estudo da maturação da gônoda feminina do "camarão rosa" (Penaeus paulensis Pérez-Farfante, 1967). Bolm Inst. Pesca, 1(4):23-38.

ZUCKERMAN, S. 1962. The ovary. London, Academic Press.

(Recebido em 24/outubro/1974) 